\title{
INFRARED DETECTORS - NEW TRENDS
}

\section{J. Piotrowski}

\author{
VIGO SYSTEM Ltd, Hery 23, 01-493 Warszawa, P.O.B. 45, Poland
}

\begin{abstract}
Recent trends in infrared detectors are towards large, electronically addressed two-dimensional arrays and higher operating temperature. This will lead both to higher performance and to smaller, lighter and more affordable IR systems. Cooling requirements are the main obstacle to the more widespread use of infrared systems based on semiconductor photodetectors, particularly in the civil field. Fundamental limitations to performance of IR photodetectors are due to the statistical nature of generation-recombination processes and resulting noise. Ways to overcome the limitations are discussed including use of the optical immersion and optical resonant cavity. Finally, the progress in a mode of operation is presented, in which the thermal generation of carriers is suppressed by the use of stationary depletion of narrow gap semiconductors. The practical near room temperature narrow gap semiconductor photodetectors are reported. Competitive technologies based on Schottky barrier devices and low dimensional solids are considered briefly. PACS numbers: 78.30.-j
\end{abstract}

\section{Introduction}

The infrared photodetectors are typically divided into two principally different groups: the thermal detectors and the photodetectors. The thermal detectors are typically operated at ambient temperature. They are unselective, slow and of low sensitivity but cheap and convenient to use. Currently, there is a considerable interest for 2D arrays of thermal detectors for low cost thermal imagers, in which the moderate sensitivity can be compensated by a large number of elements. The $240 \times 336$ arrays of $50 \mu \mathrm{m}$ pitch microbolometers have been demonstrated [1]. The devices were fabricated using the standard silicon technology. An average noise equivalent temperature difference (NETD) was better than $0.05 \mathrm{~K}$.

Fast and sensitive detection of infrared radiation can be achieved by photodetectors. Typically, photodetectors are operated at low temperature. This is necessary to prevent the thermal generation of charge carriers. The thermal transitions compete with the optical ones, making non-cooled devices very noisy.

The $\mathrm{A}^{3} \mathrm{~B}^{5}$ binary and ternary compounds are typically used for short $(>3 \mu \mathrm{m})$ applications. $\mathrm{Hg}_{1-x} \mathrm{Cd}_{x} \mathrm{Te}$ is the most important semiconductor material for infrared detectors used for devices operated the middle and long wavelength spectral range (3-25 $\mu \mathrm{m})$. A number of reviews on this material and related 
photodetectors have been published [2-6]. (See also each year Proceedings of US Workshops on Physics and Chemistry of Mercury Cadmium Telluride, 1981-1993 published in J. Vac. Sci. Technol. and J. Electron. Mater., Proceedings of 1-6 Int. Conf. on II-VI Compounds, published in J. Cryst. Growth by North-Holland, Amsterdam and Proceedings of SPIE).

Some alternative narrow gap semiconductors are recently investigated including $\mathrm{Hg}_{1-x} \mathrm{Mn}_{x} \mathrm{Te}, \mathrm{Hg}_{1-x} \mathrm{Zn}_{x} \mathrm{Te}, \mathrm{InAs}_{1-x} \mathrm{Sb}_{x}$, and other alloy systems [7]. The main motivation is to improve the material stability. No significant fundamental advantages in the ultimate performance are expected from these new materials. One important advantage of $\mathrm{Hg}_{1-x} \mathrm{Cd}_{x} \mathrm{Te}$ over the competing materials is the constant lattice parameter. This enables one to obtain perfect heterostructures with complicated and large gap variations. Such structures are important for high performance devices. It seems that the role of $\mathrm{Hg}_{1-x} \mathrm{Cd}_{x} \mathrm{Te}$ will remain unchallenged for a long time, at least for the highest quality thermal imaging.

Another competitive technologies, important for 2D arrays, are the Schottky barrier devices [8] and the quantum well infrared photodetectors (QWIPs) [9]. These technologies offer more matured technology of silicon and $\mathrm{A}^{3} \mathrm{~B}^{5}$ compounds and good performance at low temperature of operation.

This paper is mostly concentrated on new ways to improve the performance of photodetectors operated at near room temperature. The room temperature operation or at least the operation at temperatures achievable with solid state coolers is a necessary condition for more wide spread use of infrared technology in civilian and military applications.

\section{Ultimate performance of photodetectors}

Consider a photodetector as a narrow gap semiconductor flake with thickness $t$ and actual area $A_{\mathrm{e}}$. The device optical area $A_{0}$ is usually equal to its actual "electrical" area $A_{\mathrm{e}}$, but may be made different, for example by the use of an optical concentrator, as shown in Fig. 1. The generation-recombination limited detectivity is $[4-6]$

$$
D^{*}=\frac{\lambda}{h c}\left(\frac{A_{\mathrm{o}}}{A_{\mathrm{e}}}\right)^{1 / 2} \frac{\eta}{t^{1 / 2}}[2(G+R)]^{-1 / 2},
$$

where $\lambda$ is the wavelength, $h$ is the Planck constant, $c$ is the light velocity, $q$ is the electron charge, $t$ is the thickness, $h$ is the quantum efficiency, $G$ and $R$ are the generation and recombination rates. Frequently, the generation and recombination rates are equal. This situation occurs in conventional photoconductors. In this case

$$
D^{*}=\frac{\lambda}{2 h c}\left(\frac{A_{\mathrm{o}}}{A_{\mathrm{e}}}\right)^{1 / 2} \frac{\eta}{t^{1 / 2}} G^{-1 / 2} .
$$

It should be noted that the effect of recombination can be frequently avoided by arranging for the recombination process to take place in a region of the device where it has little effect due to low photoelectric gain: for example, at the contacts in sweep-out photoconductors or in the neutral regions of the photodiodes. In this case, the noise can be reduced by a factor of $2^{1 / 2}$. The generation process with its associated fluctuation, however, cannot be avoided by any means. 


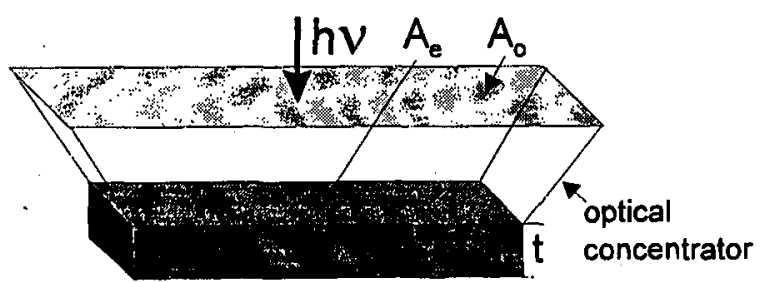

Fig. 1. The model of photodetector.

\subsection{Background limited photodetector}

Let us consider the ideal situation, when the optical generation is much stronger compared to the optical ones. The generation rate due to the optical excitation is

$$
G_{\mathrm{o}}=\frac{\Phi \eta A_{\mathrm{o}}}{t A_{\mathrm{e}}}
$$

where $\Phi$ is the photon flux. Assuming $G=R$

$$
D^{*}=\frac{\lambda}{2 h c} \frac{\eta^{1 / 2}}{\Phi^{1 / 2}} \text {. }
$$

When the recombination does not contribute to the total noise

$$
D^{*}=\frac{\lambda}{2^{1 / 2} h c} \frac{\eta^{1 / 2}}{\Phi^{1 / 2}} \text {. }
$$

If the photon flux is due to the background radiation, the expressions (4-5) become the well-known expressions for the background limited photoconductor and the background limited photodiode, respectively. It should be noted that the background limited detectivity depends no more on the $A_{\mathrm{o}} / A_{\mathrm{e}}$ ratio and on the device thickness. In further calculations, we will consider the case, when the thermal generation dominates.

\subsection{Generation-recombination mechanisms in narrow gap semiconductors}

The generation-recombination processes in semiconductors are widely discussed in Ref. [10-11]. We reproduce here only some dependencies directly related to the performance of photodetectors and concentrating on specific features of $\mathrm{Hg}_{1-x} \mathrm{Cd}_{x} \mathrm{Te}$.

Assuming bulk processes only, there are three main thermal generationrecombination processes to be considered in the narrow band gap semiconductors: Shockley-Read, radiative and Auger.

The Shockley-Read mechanism is not an intrinsic and fundamental process as it occurs via levels in forbidden energy gap. It may be reduced by lowering concentrations of native defects and foreign impurities. This can be achieved in low temperature growth and by progress in purification of materials. Though still a considerable research effort is necessary, the Shockley-Read process does not represent a fundamental limit to performance of the photodetectors. 
The radiative generation-recombination and Auger mechanisms ere fundamental band-to-band processes which are determined by the electronic band structure of the semiconductor. The role of radiative mechanism in detection of IR radiation has been recently critically reexamined by Humpreys [12]. He indicated that most of photons emitted in photodetectors as a result of a radiative decay is immediately re-adsorbed, so that the observed radiative lifetime is only a measure how well photons can escape from the body of detector. Due to re-absorption the radiative lifetime is highly extended, in dependence of the semiconductor geometry. The fundamental limit to the detector performance is reached, when the radiation generation is principally caused by the photons from the scene, while the secondary recombination-generation processes are practically noiseless.

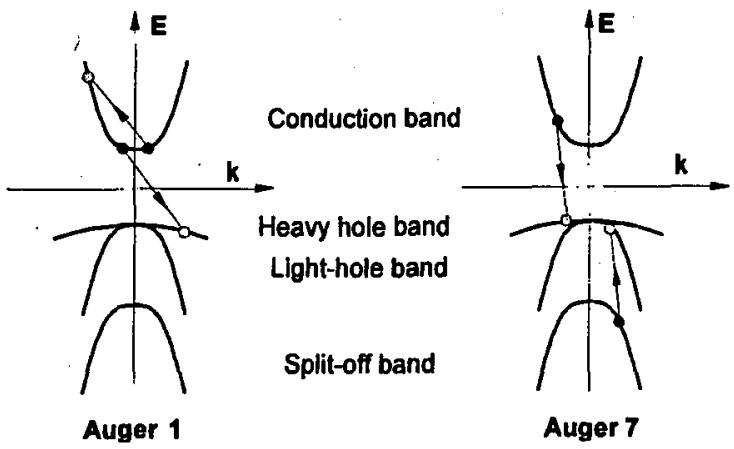

Fig. 2. The two most important band-to-band Auger recombination processes. Arrow shows the electron transitions.

The Auger mechanism dominates generation and recombination processes in high quality $\mathrm{InSb}$ and narrow gap $\mathrm{Hg}_{1-x} \mathrm{Cd}_{x} \mathrm{Te}$ [11]. The band-to-band Auger effects are classified in several processes according to related bands. Ten types of photonless Auger recombination mechanisms are possible in a semiconductor with a single conduction band and heavy- and light-hole valence bands. Figure 2 shows the two most important mechanisms in the case of this type of band structure. They have the smallest threshold $\left(E_{\mathrm{T}} \approx E_{\mathrm{g}}\right)$ and have the largest combined density of states. The Auger 1 recombination mechanism involves two electrons and a heavy hole and is dominant in $n$-type material. The Auger 7 process is dominant in $p$-type material if the spin split-off band can be ignored. For a material such as InSb and $\mathrm{Hg}_{1-x} \mathrm{Cd}_{x} \mathrm{Te}$, where the spin split-off energy $\Delta$ is much larger than the band gap energy $E_{\mathrm{g}}$, the probability of the Auger transition through the conduction band-heavy hole band-spin split-off band mechanism may be negligibly small in comparison with that of the Auger 7 transition.

The generation and recombination rates due to the Auger 1 and Auger 7 processes can be described as

$$
G_{\mathrm{A}}=\frac{n}{2 \tau_{\mathrm{Al}}^{\mathrm{i}}}+\frac{p}{2 \tau_{\mathrm{A} 7}^{\mathrm{i}}},
$$




$$
R_{\mathrm{A}}=\frac{n p}{2 n_{\mathrm{i}}^{2}}\left(\frac{n}{\tau_{\mathrm{A} 1}^{\mathrm{i}}}+\frac{p}{\tau_{\mathrm{A} 7}^{\mathrm{i}}}\right)
$$

where $\tau_{\mathrm{A} 1}^{\mathrm{i}}$ and $\tau_{\mathrm{A} 7}^{\mathrm{i}}$ are the intrinsic Auger 1 and Auger 7 recombination times, $n_{\mathrm{i}}$ is the intrinsic concentration. The last equation is valid for a wide range of concentrations. The Auger 1 and Auger 7 intrinsic times are

$$
\tau_{\mathrm{A} 1}^{\mathrm{i}}=\frac{3.8 \times 10^{18} \varepsilon_{\infty}^{2}(1+\mu)^{1 / 2}(1+2 \mu) \exp \left[\left(\frac{1+2 \mu}{1+\mu}\right) \frac{E_{\mathrm{g}}}{k T}\right]}{\left(m_{\mathrm{e}}^{*} / m\right)\left|F_{1} F_{2}\right|^{2}\left(k T / E_{\mathrm{g}}\right)^{3 / 2}},
$$

where $\mu$ is the ratio of the conduction to the heavy-hole valence-band effective mass, $\varepsilon_{\infty}$ is the high frequency dielectric constant and $\left|F_{1} F_{2}\right|$ are the overlap integrals of the periodic part of the electron wave functions. Just the overlap integrals cause the biggest uncertainly in the Auger 1 lifetime. Values of ranging from 0.1 to 0.3 have been obtained by various authors. In practice it is taken as a constant equal to anywhere between 0.1 and 0.3 leading to changes by an order of magnitude in the lifetime.

The ratio of Auger 7 and Auger 1 intrinsic times

$$
\gamma=\tau_{\mathrm{A} 7}^{\mathrm{i}} / \tau_{\mathrm{A} 1}^{\mathrm{i}}
$$

is another term of high uncertainty. According to Ref [13], for $\mathrm{Hg}_{1-x} \mathrm{Cd}_{x} \mathrm{Te}$ over the range $0.16 \leq x \leq 0.40$ and $50 \leq T \leq 300 \mathrm{~K}, 3 \leq \gamma \leq 6$. As $\gamma$ is higher than unity, higher recombination lifetimes are expected in lightly doped $p$-type materials.

\subsection{Equilibrium mode}

At equilibrium the generation and recombination rates are equal. Let us consider the Auger limited detectivity. For non-generated statistics

$$
G_{\mathrm{A}}=\frac{n}{2 \tau_{\mathrm{A} 1}^{\mathrm{i}}}+\frac{p}{2 \tau_{\mathrm{A} 7}^{\mathrm{i}}}=\frac{1}{2 \tau_{\mathrm{A} 1}^{\mathrm{i}}}\left(n+\frac{p}{\gamma}\right)
$$

The Auger dominated detectivity is

$$
D^{*}=\frac{\lambda}{2^{1 / 2} h c}\left(\frac{A_{\circ}}{A_{\mathrm{e}}}\right)^{1 / 2} \frac{\eta}{t^{1 / 2}}\left(\frac{\tau_{\mathrm{A} 1}^{\mathrm{i}}}{n+p / \gamma}\right)^{1 / 2} .
$$

The resulting Auger generation achieves its minimum for $p=\gamma^{1 / 2} n_{\mathrm{i}}$. This leads to important conclusion about optimum doping for the best performance. Since $\gamma>1$ the highest detectivity of Auger limited photodetectors can be achieved with $p$-type doping. In practice, the required $p$-type doping level would be difficult to achieve in practice. At low temperature the $p$-type devices suffer from some non-fundamental limitations (contacts, surface, Shockley-Read processes) more than the $n$-type ones. This is the reason why low temperature photodetectors are typically manufactured from lightly doped $n$-type materials. In contrast, $p$-type doping is clearly advantageous for the near room temperature and long wavelength photodetectors [13]. 
At near room temperature the electron and hole concentrations in narrow gap semiconductors are equal to the intrinsic concentration. The decrease in temperature strongly increases $\tau_{\mathrm{A} 1}^{\mathrm{i}}$ and decreases the intrinsic concentration sharply improving $D^{*}$. At lower temperatures when the concentration of majority of carriers saturates at an extrinsic level, the performances still improve with decreasing of temperature due to increasing of $\tau_{\mathrm{A} 1}^{\mathrm{i}}$.

Assuming here that the optical and actual areas of a photodetector are equal, the detectivity is determined by $\eta /(t G)^{1 / 2}$, which is the ration of quantum efficiency to the number of generation acts per unit of area. The $\eta$ and $G$ terms are not independent, however. The increase in band gap strongly reduces the Auger generation rate, but it may drastically reduce the quantum efficiency due to decreasing absorption. To optimize the performance the $\eta /(t G)^{1 / 2}$ product has to be maximized as a function of gap and doping.

To estimate the Auger limited detectivity of photodetectors operated at various wavelengths let us assume that the optimum performance can be achieved for a band gap close to the energy of photons to be detected. The assumption is actually based on the sharp dependence of the absorption coefficient on wavelength near to the fundamental edge of absorption. Next, let us assume that the quantum efficiency of $10 \mu \mathrm{m}$ thick photodetectors prepared from $E_{\mathrm{g}}=h c / \lambda$ material is equal to 0.5 . Detailed calculations based on full optimization of $D^{*}\left(E_{\mathrm{g}}\right)$ and those on above assumptions agree within a factor of 2 in the range of $2<\lambda<20 \mu \mathrm{m}$ and $77<T<300 \mathrm{~K}[6]$.

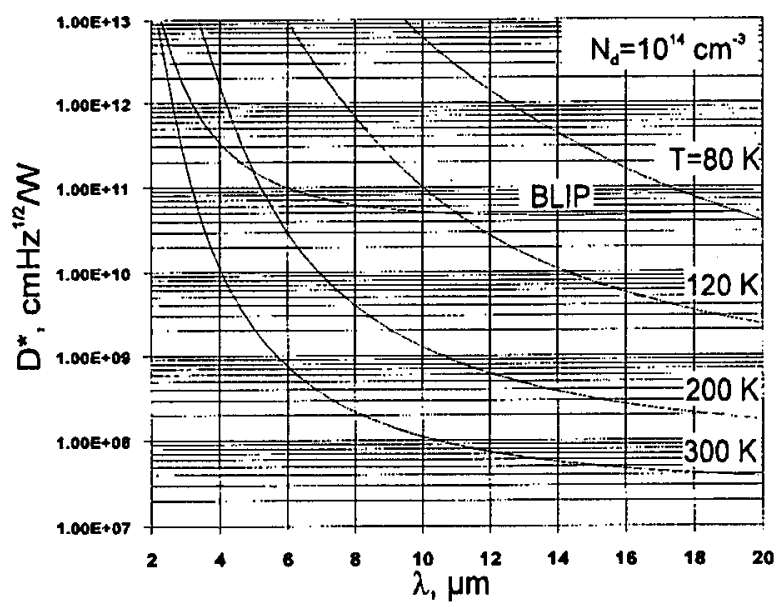

Fig. 3. Calculated performance of generation-recombination limited $\mathrm{Hg}_{1-x} \mathrm{Cd}_{x} \mathrm{Te}$ photodetectors as a function of wavelength and temperature of operation. BLIP detectivity has been calculated for $2 \pi \mathrm{FOV}, T_{\mathrm{b}}=300 \mathrm{~K}, \eta=1$.

Figure 3 shows calculated detectivity of generation-recombination limited $\mathrm{Hg}_{1-x} \mathrm{Cd}_{x} \mathrm{Te}$ photodetectors as a function of wavelength and temperature of operation. The calculation has been performed for $10^{14} \mathrm{~cm}^{-3}$ donor doping, which 
is the lowest doping achievable in controllable manner in practice. In principle, the liquid nitrogen cooling makes possible to achieve BLIP (background limited infrared detector) performance in the $2-18 \mu \mathrm{m}$ range. $200 \mathrm{~K}$ cooling, which is achievable with Peltier coolers, would be sufficient for BLIP operation in the middle and short wavelength regions $(<5 \mu \mathrm{m})$.

\subsection{Non-equilibrium mode}

British workers [14-18] proposed a new approach to reduce the photodetector cooling requirements, which is based on the non-equilibrium mode of operation. Their concept relies on suppression of the Auger processes by decreasing the free carrier concentration below its equilibrium values. At strong depletion the concentration of both majority and minority carriers can be reduced below intrinsic concentration. The necessary condition is a very light doping of semiconductor, since at strong depletion the majority of carrier concentrations saturates on the extrinsic level. In contrast, the concentration of minority of carries is reduced below the extrinsic level. The depletion can be achieved, for example, in a biased $l-h$ or heterojunction contact devices or using the magnetoconcentration effect $[5,19]$.

Let us consider lightly doped $n$-type material ( $\nu$-type) with a donor concentration $N_{\mathrm{d}}\left(N_{\mathrm{d}} \ll n_{\mathrm{i}}\right)$. At strong depletion $n=N_{\mathrm{d}}$ and the Auger generation rate is

$$
G_{\mathrm{A}}=\frac{N_{\mathrm{d}}}{2 \tau_{\mathrm{A} 1}^{\mathrm{i}}} \text {. }
$$

Depletion makes the recombination rate negligible compared to the generation rate. In this case

$$
D^{*}=\frac{\lambda}{h c}\left(\frac{A_{\mathrm{o}}}{A_{\mathrm{e}}}\right)^{1 / 2} \frac{\eta}{t^{1 / 2}}\left(\frac{\tau_{\mathrm{A} 1}^{\mathrm{i}}}{N_{\mathrm{d}}}\right)^{1 / 2}
$$

Similarly, for lightly doped $p$-type

$$
\begin{aligned}
& G_{\mathrm{A}}=\frac{N_{\mathrm{a}}}{2 \gamma \tau_{\mathrm{A} 1}^{\mathrm{i}}}, \\
& D^{*}=\frac{\lambda}{h c}\left(\frac{A_{\circ}}{A_{\mathrm{e}}}\right)^{1 / 2} \frac{\eta}{t^{1 / 2}}\left(\frac{\tau_{\mathrm{A} 1}^{\mathrm{i}}}{N_{\mathrm{a}} / \gamma}\right)^{1 / 2} .
\end{aligned}
$$

Again, as in the equilibrium case, the use of p-type material is advantageous, improving the detectivity by a factor of $\gamma^{1 / 2}$ compared to $n$-type material of the same doping. Comparing the corresponding equations for equilibrium and non-equilibrium modes, we can find that the use of non-equilibrium mode of operation can reduce the Auger generation rate by a factor $n_{\mathrm{i}} / N_{\mathrm{d}}$ with the corresponding improvement of detectivity by $\left(2 n_{\mathrm{i}} / N_{\mathrm{d}}\right)^{1 / 2}$. The additional gain factor of $2^{1 / 2}$ is due to a negligible recombination rate in depleted semiconductor. This improvement may be quite large, particularly for long wavelenght infrared (LWIR) devices operated near to room temperatures as shown in Fig. 4. In principle, the BLIP performance can be obtained without cooling at all. This can be achieved, however, by the use of extremely high quality materials, with a very low concentration of the shallow and Shockley-Read centers. Utmost care has also to be taken with a contact and passivation technology. In this respect, the heterostructure passivation and contacts are strategically important. 


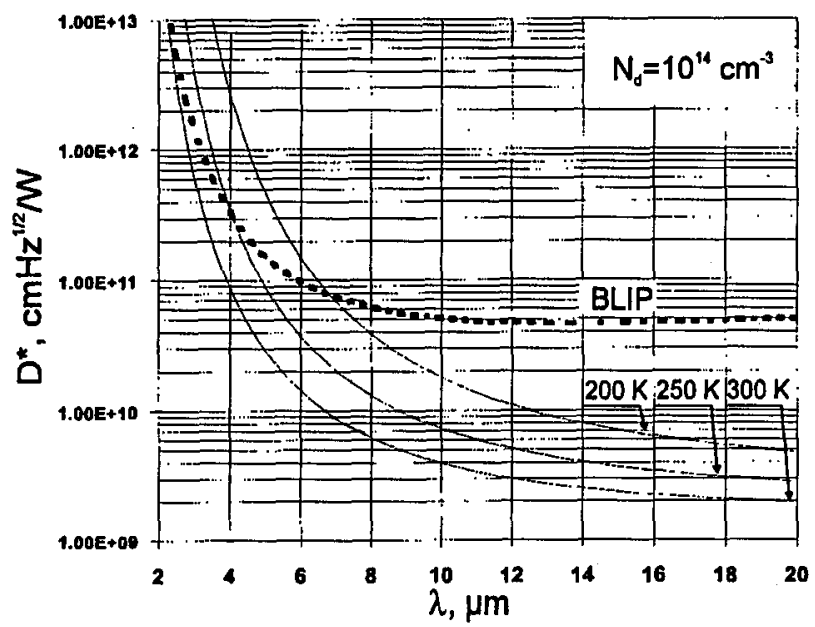

Fig. 4. Calculated performance of generation-recombination limited and operated in the non-equilibrium mode $\mathrm{Hg}_{1-x} \mathrm{Cd}_{x} \mathrm{Te}$ photodetectors as a function of wavelength and temperature. BLIP detectivity has been calculated for $2 \pi \mathrm{FOV}, T_{\mathrm{b}}=300 \mathrm{~K}$ and $\eta=1$.

\subsection{Reduced volume devices}

Another way to improve the performance is to reduce the volume of semiconductor by reduction of the detector thickness and its actual area. This must be done without a decrease in quantum efficiency and preserving the required optical area of a detector.

The $\eta / t^{1 / 2}$ term. Since the detectivity is proportional to the factor $\eta / t^{1 / 2}$, high quantum efficiency must be achieved in thin devices. This is usually difficult to realize in practice for devices operated at near room temperature, as the absorption of long-wavelength radiation in narrow gap semiconductors is weak for a band gap close to the photon energy.

The quantum efficiency can be enhanced in thin devices with non-reflective frontside and highly reflective backside surfaces of the sensitive element. This can be achieved in a multilayer structure, shown in Fig. $5 \mathrm{a}$, in which the semiconductor flake is sandwiched between two dielectric layers and a backside metal reflecting mirror. The effective absorption can be improved further using interference effect (see Ref. [4-6] and related papers cited therein) to set up the resonant cavity within the photodetector. The optimized resonant cavity structure with carefully selected thickness and reflection coefficients of all layers exhibit detectivity enhanced by a factor of about 2.5 at peak wavelength compared to the optimized conventional $10.6 \mu \mathrm{m}$ devices. It should be noted that the interference effects strongly modify the spectral response of the device, and the gain due to optical cavity can be achieved only in narrow spectral regions.

The $A_{\mathrm{o}} / A_{\mathrm{e}}$ term. A possible way to achieve an effective concentration of radiation is to immerse the photodetector to the hemispherical of hyperhemispherical lenses of a high refraction coefficient (Fig. 5b) [3-6, 20-22]. Due to immersion, the 


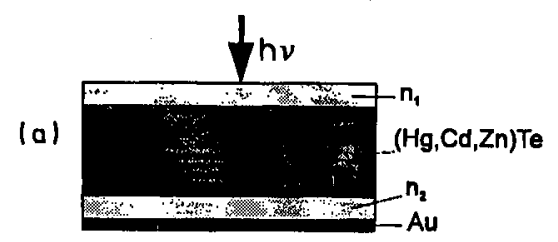

(b)

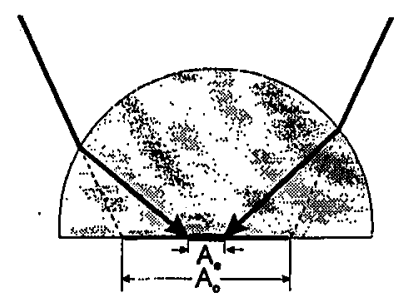

Fig. 5. Schematic structure of reduced volume photodetectors: (a) enhanced quantum efficiency device, (b) optically immersed detector.

linear size of the detector increases by a factor of $n$ or $n^{2}$ for hemispherical and hyperhemispherical lens, respectively ( $n$ is the refraction coefficient). As a result, the detectivity is increased by the same factor. Germanium $(n=4)$ is the most frequently used for immersion lens. The practical use of immersion technology has been limited due to problems in mechanical matching of detector and lens material, severe transmission and reflection losses. Another limitation was due to a limited acceptance angle of the devices as a result of the total reflection at the lens-glue interface.

The problems have been recently solved by the use of monolithic technology developed at VIGO [4-6]. The technology was based on epitaxy of $\mathrm{HgCdZnTe}$. The $\mathrm{HgCdZnTe}$ serves as the sensitive element, while the immersion lens is formed directly in the transparent $(n=2.7)$ substrate.

As Table shows, the monolithic optical immersion results in significant improvement of detector parameters. The gain factors achieved with hyperhemispherical immersion are substantially higher compared to those for hemispherical immersion. The hyperhemispherical immersion may restrict the acceptance angle of the detector and require more severe manufacturing tolerances. These restrictions depend on the refraction coefficient of the lens. For $(\mathrm{Cd}, \mathrm{Zn}) \mathrm{Te}$ they are not so severe as for germanium lenses, however, and have no practical importance in most cases. For example, the numerical aperture of the main optical system is limited to about 1.4 by the immersion lens.

Some gains due to optical immersion are specific for particular types of photodetector. For example, the reduction of bias power dissipation is important in case of photoconductors and these photodiodes which require strong bias for the best performance. The immersion also significantly reduces the heat load of heat sinks and cooling devices, making possible to reduce size, weight, price and power consumption of cooling systems. The reduction of capacitance due to immersion 
TABLE

Influence of optical immersion on properties of photodetectors with $\mathrm{CdZnTe}$ lens. The numbers show the relative change of a given value compared to the non-immersed detector of the same optical area.

\begin{tabular}{l|lr|lr|c}
\hline \hline \multicolumn{1}{c|}{ Value } & \multicolumn{2}{|c|}{ Hemisphere } & Hyperhemisphere & Type of detector \\
\hline Linear size & $n$ & $(=2.7)$ & $n^{2}$ & $(=7)$ & any \\
Area & $n^{2}$ & $(=7)$ & $n^{4}$ & $(=49)$ & any \\
Voltage responsivity & $n$ & $(=2.7)$ & $n^{2}$ & $(=7)$ & PC, PEM \\
Voltage responsivity & $n^{2}$ & $(=7)$ & $n^{4}$ & $(=49)$ & PV \\
Detectivity & $n$ & $(=2.7)$ & $n^{2}$ & $(=7)$ & any \\
Bias power & $n^{2}$ & $(=7)$ & $n^{4}$ & $(=49)$ & PC, PV \\
Capacitance & $n^{2}$ & $(=7)$ & $n^{4}$ & $(=49)$ & PV \\
Acceptance angle, deg & & 180 & & 42 & any \\
Thickness/radius & & 1 & & stringent & any \\
Tolerances & not serve & & &
\end{tabular}

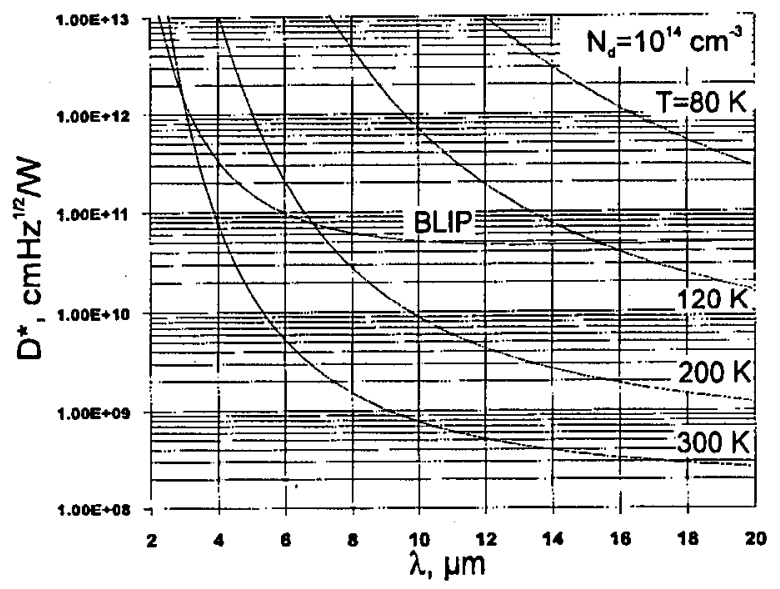

Fig. 6. Calculated performance of optically immersed generation-recombination limited $\mathrm{Hg}_{1-x} \mathrm{Cd}_{x} \mathrm{Te}$ photodetectors as a function of wavelength and temperature. $\mathrm{Hy}-$ perspherical immersion, lens refraction coefficient $n=2.7$. BLIP detectivity has been calculated for $2 \pi$ FOV, $T_{\mathrm{b}}=300 \mathrm{~K}$ and $\eta=1$.

is of utmost importance for high frequency photodiodes which makes possible to improve the high frequency performance of $\mathrm{RC}$ limited devices by a large factor.

Figure 6 shows the calculated performance of optically immersed generationrecombination limited $\mathrm{Hg}_{1-x} \mathrm{Cd}_{x} \mathrm{Te}$ photodetectors as a function of wavelength and temperature. The optical immersion brings the performance of near room temperature photodetectors much closer to the BLIP limit. Actually, the BLIP 
detectivity would be achievable in the middle wavelength infrared (MWIR) spectral range with the simple two-stage thermoelectric coolers. The combination of optical immersion and non-equilibrium mode operation can significantly soften the conditions for BLIP operation. Even with moderate $10^{14} \mathrm{~cm}^{-3}$ doping the BLIP performance should be potentially achievable at room temperature in the MWIR and with thermoelectric cooling in the LWIR spectral range.

\section{Practical devices}

Figure 7 shows two structures of practical photoconductors prepared with the use of 3D architecture of heterostructure with lightly $p$-type doped main region and heavily $n$-type doped, wider gap $\underline{n}^{+}$-type contact regions. Both structures have been prepared using isothermal vapor phase epitaxy. The growth has been carried out in the production-type reusable growth system [23] shown in Fig. 8. The band gap profiles have been shaped using selective epitaxy and variations of growth conditions. The low level $p$-type doping was obtained by doping in situ during growth with foreign impurities. The heavily $n$-type doped layer has been obtained by a shallow ion milling.

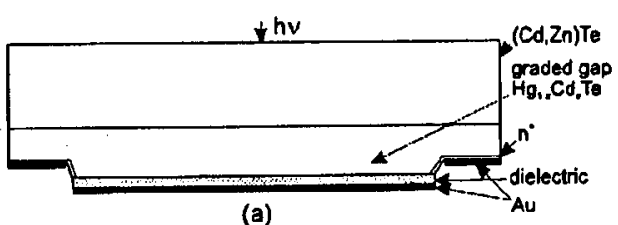

(a)

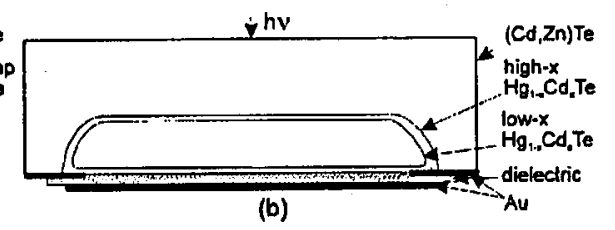

(b)

Fig. 7. Schematic of 3D heterostructure photoconductors.

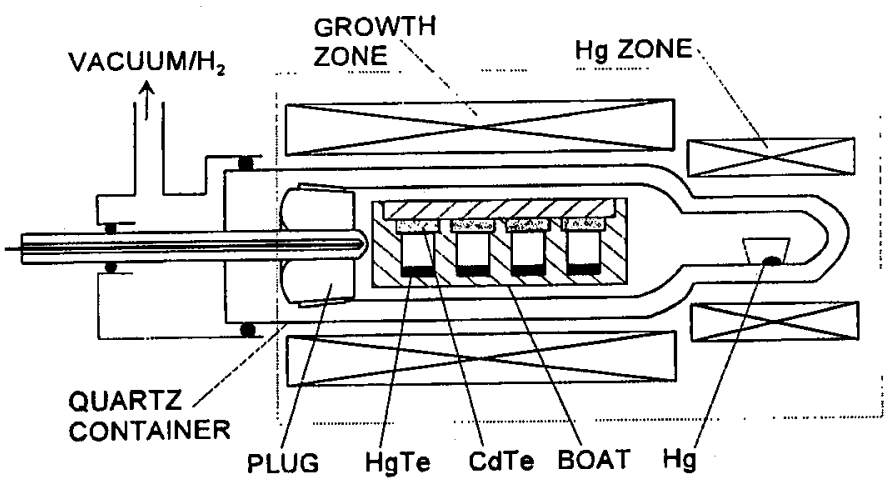

Fig. 8. Isothermal vapor phase growth reactor.

Initial efforts were concentrated mostly on structure (a), based on laterally homogeneous epilayers with in-depths gap grading. In this case, the contact areas have been prepared by a combination of the chemical and ion etching. Recent efforts are on device (b). In this approach the absorbing layer of $\mathrm{Hg}_{1-x} \mathrm{Cd} x \mathrm{Te}$ 
is buried in the graded gap layer with larger $x$-value to provide heterostructure passivation of the active area. The increase of gap at the surface has been achieved using the negative epitaxy, which is near equilibrium evaporation of HgTe from the surface of the $\mathrm{Hg}_{1-x} \mathrm{Cd}_{x} \mathrm{Te}$ layer.

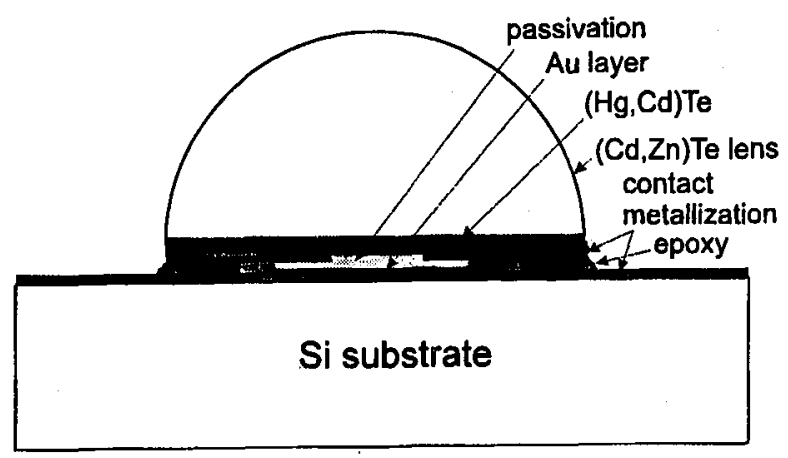

Fig. 9. Structure of advanced monolithic optically immersed photoconductor.

The devices are then optically immersed to the lenses formed directly in the transparent $\mathrm{CdZnTe}(n=2.7)$ substrate of $\mathrm{Hg}_{1-x} \mathrm{Cd}_{x} \mathrm{Te}$ layer (Fig. 9). The relatively low refraction coefficient of $\mathrm{CdZnTe}(n=2.7)$ makes the expected gain lower compared to germanium, but hyperhemispherical CdZnTe immersion lenses can be used in relatively fast optics, achieving $c a$. a 7 -fold increase in detectivity and decrease in bias power dissipation by a factor of about 50 . The lenses have diameters in the range of 0.5 to $8 \mathrm{~mm}$. The monolithic approach permits simple and economical manufacturing, the devices are rugged, mechanically stable and they can operate in a very broad spectral band with minimized reflection and absorption losses. The sensitive elments are housed in various packages, depending on their operating temperature, frequency band, active area and other factors. Some examples are shown in Fig. 10. The thermoelectrically cooled detectors are typically housed in modified TO-8 cans, supplied with IR transparent windows (AR-coated $\mathrm{Ge}$, sapphire, $\mathrm{BaF} 2, \mathrm{ZnS}$ and others). The cans are then backfilled with low thermal conductivity gas and sealed off.

Figure 11 shows spectral detectivities of ambient temperature and thermoelectrically cooled MWIR and LWIR photoconductors. The devices have configuration shown in Fig. 7a, with symmetric anode and cathode regions. The performance of the devices has been improved by a large factor compared to the conventional devices. The measured detectivity of uncooled $10.6 \mu \mathrm{m}$ photoconductors is by many orders of magnitude higher compared to other ambient temperature $10.6 \mu \mathrm{m}$ detectors with subnanosecond response time as photon drag detectors, fast thermocouples, bolometers and pyroelectric ones, approaching the performance of slow thermal detectors. The uncooled devices optimized at $\lambda \approx 9 \mu \mathrm{m}$ exhibit thermal figure of merit $M^{*}$ larger compared to that of thermal detectors. Near background limited performance has been achieved with 3-5 $\mu \mathrm{m}$ devices cooled with 2-stage TE coolers. 5-stage coolers are necessary to achieve the same in the 8-12 $\mu \mathrm{m}$ range. 

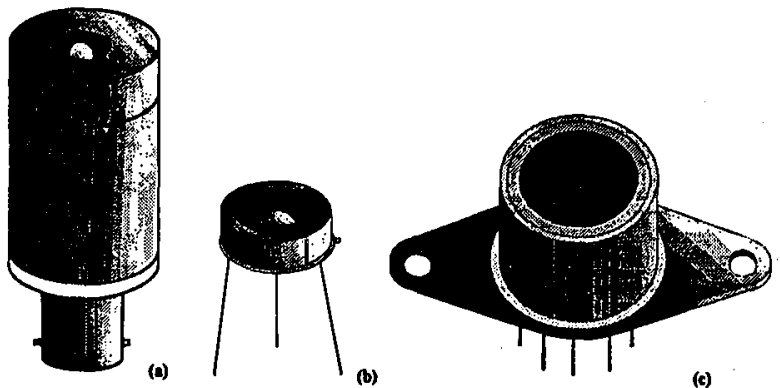

Fig. 10. Optically immersed MWIR and LWIR $\mathrm{Hg}_{1-x} \mathrm{Cd}_{x}$ Te photodetectors: (a), (b) ambient temperature, (c) Peltier cooled devices.
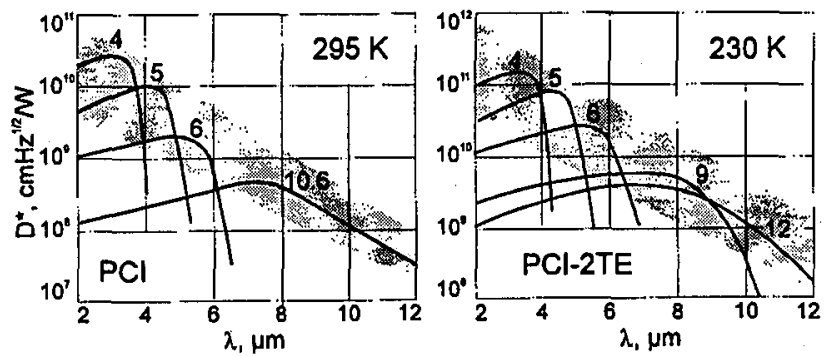

Fig. 11. Spectral detectivities $(f=20 \mathrm{kHz})$ of optically immersed $\mathrm{Hg}_{1-x} \mathrm{Cd}_{x} \mathrm{Te}$ photoconductors, uncooled and cooled with 2-stage Peltier cooler.

The resulting devices show a great promise for near-BLIP operation of MWIR and LWIR photodetectors with simple thermoelectric coolers. It should be noted that the principles of optical resonant cavity and optical immersion have been already successfully applied to photoelectromagnetic, Dember effect and photovoltaic detectors [2-6]. Comparing the measured detectivities with theoretical limits imposed by unavoidable physics one can see a significant discrepancy. This is a result of non-optimum design. It seems that the main contribution to the total noise comes from thermal generation at contact, interfaces and surface regions. The preparation of the cathode contact seems to be the most critical step.

Further efforts are underway. Encouraging results have been recently obtained for devices with an independently optimized gap and compositional profiles for the anode and cathode regions [24].

\section{References}

[1] R.A. Wood, N.A. Foss, Laser Focus World, June, 101 (1993).

[2] A. Rogalski, J. Piotrowski, Prog. Quant. Electr. 12, 87 (1988).

[3] J. Piotrowski, W. Galus, M. Grudzień, Infrared Physics 31, 1 (1990).

[4] J. Piotrowski, Optoelectronics Rev. 1, 9 (1992). 
[5] J. Piotrowski, W. Gawron, Z. Djuric, Optical Engineering 33, 1413 (1994).

[6] J. Piotrowski, in: Semiconductor Infrared Detectors, SPIE, Bellingham 1995, to be published.

[7] A. Rogalski, Liquid and Solid State Crystals, Proc. SPIE 1845, 52 (1992) and original papers cited therein.

[8] M. Kimata, N. Tubouchi, in: Semiconductor Infrared Detectors, SPIE, Bellingham 1995, to be published.

[9] F.F. Sizov, in: Semiconductor Infrared Detectors, SPIE, Bellingham, to be published.

[10] A. Rogalski, in: Semiconductor Infrared Detectors, SPIE, Bellingham, to be published.

[11] P.E. Petersen, in: Semiconductors and Semimetals, Eds. R.K. Willardson, A.C. Beer, Vol. 18, Academic Press, New York 1981, p. 121.

[12] R.G. Humpreys, Infrared Phys. 23, 171 (1983); ibid. 26, 337 (1986).

[13] D.L. Spears, in: Proc. IRIS Active Systems, San Diego 27-30 July 1982, (1982).

[14] T. Ashley, C.T. Elliott, A.M. White, in: Infrared Technology, SPIE 572, 123 (1985).

[15] T. Ashly, C.T. Elliott, A.T. Harker, Infrared Phys. 26, 303 (1986).

[16] A.M. White, J. Cryst. Growth 86, 840 (1988).

[17] C.T. Elliott, Semicond. Sci. Technol. 5, S30 (1990).

[18] T. Ashly, T.C. Elliott, Semicond. Sci. Technol. 8, C99 (1991).

[19] Z. Djuric, Z. Jaksic, A. Vujanic, J. Piotrowski, J. Appl. Phys. 71, 5706 (1992).

[20] J.E. Slavek, H.H. Randal, Infrared Phys. 15, 339 (1975).

[21] I.C. Carmichael, A.B. Dean, D.J. Wilson, Advanced Infrared Detector and Systems, IEE London 1983, p. 45.

[22] M. Grudzień, J. Piotrowski, Infrared Phys. 29, 251 (1989).

[23] J. Piotrowski, Z. Nowak, M. Grudzień, W. Galus, K. Adamiec, Z. Djurić, V. Jović, Z. Djinović, Thin Solid Films 161, 157 (1988).

[24] J. Piotrowski, to be published. 